\title{
LA RELACIÓN ENTRE ABUELOS Y NIETOS Factores que predicen la calidad de la relación intergeneracional ${ }^{*}$
}

\author{
SACRAMENTO PINAZO \\ y JULIÁN MONTORO \\ Universidad de Valencia y Kent State University, Kent, Ohio. USA
}

\author{
PALABRAS CLAVE ADICIONALES \\ ADDITIONAL KEYWORDS \\ Relaciones intergeneracionales, satisfacción \\ Intergenerational Relations, Satisfaction
}

RESUMEN. El presente estudio se centra en la identificación de los factores que predicen la calidad en la relación intergeneracional abuelos-nietos desde la percepción que tienen los jóvenes. Los resultados de nuestra investigación se basan en una muestra de 361 jóvenes españoles entre 15 y 25 años que respondieron a un cuestionario sobre el "abuelo preferido". Los resultados del análisis multivariante indican que los aspectos que determinan la calidad de la relación son: a) la frecuencia del contacto entre abuelos y nietos; b) la relación cercana y frecuente entre abuelos y padres; c) las actividades de acompañamiento (conversar, pasear, mirar fotos y recuerdos familiares, visitar amigos y familiares, discutir y tomar decisiones, rezar) entre abuelos y nietos; y d) la percepción del abuelo/a como cuidador y como maestro, fuente de adquisición de conocimientos y destrezas.

ABSTRACT. This study tries to identify the main factors determining the quality in grandparent/ grandchild relationships from the perspective of grandchildren. Three hundred and sixty one Spanish young adults responded to questions concerning the quality of their relationship with the "preferred" or "most close" grandparent. Results indicated the following aspects as significant predictors of grandparent-grandchild relationship: a) the frequency of contact between grandparents-grandchildren; b) the quality of the parent-grandparent relationship; c) the number of shared activities; and d) the perception of the grandparent as "caregiver" and "teacher".

\footnotetext{
E-mail: sacramento.pinazo@uv.es 02604)

*Esta investigación forma parte del Proyecto I+D financiado por la DIGICYT (BSO2002-
}

Revista Internacional de Sociología (RIS)

Tercera Época, No 38, Mayo-Agosto, 2004, pp. 147-168. 
R I S

REVISTA INTERNACIONAL DE SOCIOLOCIA

№ 38, MAYO-ACOSTO, 2004

SACRAMENTO PINAZO Y JULLÁN MONTORO

\section{INTRODUCCIÓN}

El estudio de las relaciones abuelos-nietos se ha desarrollado considerablemente desde los años 80 en los países anglosajones debido a diversos motivos: entre otros, el cambio demográfico operado durante la mitad del siglo XX y, sobre todo, el aumento progresivo de personas mayores como consecuencia del proceso de envejecimiento en las sociedades industriales. El aumento de los mayores de 65 años proporciona la oportunidad para una mayor presencia y participación de los abuelos en las decisiones y responsabilidades del sistema familiar. Las consecuencias de estos cambios sociodemográficos se pueden apreciar en diversos aspectos de las relaciones familiares: en particular, en el incremento en la duración de las relaciones intergeneracionales y del número de abuelos que ven a sus nietos alcanzar la adolescencia, la juventud e incluso la edad adulta. Iguẩlmente, la existencia de relaciones de larga duración entre los abuelos y nietos, así como su incorporación activa en tareas como el cuidado de los nietos (Tobío y Fernández-Cordón, 1999) son cambios que indican la relevancia cada vez mayor de la figura del abuelo en el sistema familiar. Las relaciones intergeneracionales y los vínculos que los abuelos establecen con sus nietos son aspectos importantes que están siendo objeto de estudio por los investigadores sociales. Los estudios reconocen de forma especial los beneficios que la figura del abuelo puede aportar al desarrollo global y al proceso de socialización de los nietos, poniendo de relieve los aspectos que son más propios del carácter especial de las relaciones nietos-abuelos desde la perspectiva de los abuelos y de los nietos (Kennedy, 1992a; Williams y Nussbaum, 2001). La presente investigación se centra en la identificación de los factores que contribuyen a incrementar la calidad de las relaciones intergeneracionales entre los abuelos y los nietos.

Según el estudio 2072 del CIS-IMSERSO (IMSERSO, 1995), entre los mayores de 65 años con hijos, el 35\% presta algún tipo de ayuda a éstos en tareas domésticas o en el cuidado de los nietos. La mitad de los mayores desempeñan un rol asistencial activo si se encuentran en circunstancias personales (capacidad) o situacionales (cercanía o convivencia) apropiadas para ello. Por ejemplo, entre los que declaran valerse por sí mismos, tener hijos y salud buena o regular, ayudan a sus hijos o nietos entre el $35 \%$ y $40 \%$. Obviamente, la tasa de colaboradores en las tareas domésticas de sus hijos o en el cuidado de los nietos es más alta cuando el anciano convive con ellos bajo el mismo techo (44\%) que en otras circunstancias. Pero incluso cuando la persona mayor vive sola, es frecuente que ayude a sus hijos o nietos (26\%). La ayuda intergeneracional focalizada en el cuidado de los nietos y en tareas domésticas se da en mayor proporción entre mujeres (40\%) que entre hombres $(29 \%)$. Con respecto al tipo de ayuda suministrada a los nietos, ocupan el primer puesto las tareas de cuidado. Poco más de uno de cada diez realiza cuidados habituales diarios como dar de comer a los nietos o acompañarlos al ir o volver del colegio. Según los datos del Informe 2000: Las personas mayores 
en España (Sancho, 2000), aproximadamente ocho de cada diez tienen nietos y los ven con mucha frecuencia. El $61 \%$ de los mayores tiene contactos con nietos al menos varias veces al mes, y el 52\% habla con ellos por teléfono con esa frecuencia, además de que el grado de satisfacción de las relaciones es muy elevado, puesto que el $95 \%$ se considera muy o bastante satisfecho. En España, aunque escasos, los estudios realizados sobre la relación abuelos-nietos se han centrado en la percepción de las abuelas (Benlloch y Berjano, 1994; Benlloch, Calatayud, Llopis, Pinazo y Berjano, 1994) o de las abuelas y los abuelos (Triadó y Villar, 2000) y en la percepción de los nietos durante la infancia (Rico, 2000; Rico, Serra y Viguer, 2001), pero pocos han estudiado a los nietos durante la adolescencia (Triadó, Martínez y Villar, 1999), la juventud o la adultez.

Algunos autores han calificado la relación actual entre los ancianos y sus familias con el título de intimidad a distancia, término acuñado por Rosenmayr en 1977. Bajo este nombre se reconoce la naturaleza personalísima y única de la relación a través del término intimidad, pero se afirma también que entre ambas partes se respeta la independencia y por ello debe existir cierta autonomía o distancia que desaparece cuando la persona mayor tiene una necesidad concreta (salud, compañía, etc.) y/o asume el cuidado directo de los jóvenes cuando los padres están ausentes (cuidador de los nietos, etc.). Otros estudios han examinado la percepción de la relación abuelos-nietos preguntando a nietos adultos (Robertson, 1976; Hoffman, 1979; Hartshorne y Manaster, 1982; Eisenberg, 1988). En 1994 el CIREM realizó un estudio intergeneracional con una muestra formada por 1.250 sujetos de dos grupos de edad (uno de 65-74 años y otro de 14-17 años). Los datos mostraron que más de un $65 \%$ de las personas mayores con nietos de estas edades afirman tener contactos regulares con ellos, para conversar, darles dinero o regalarles cosas; el $50 \%$ manifiesta que la comunicación se establece por teléfono y uno de cada diez hace referencia a actividades conjuntas, como hacer encargos (CIREM, 1994).

El período del final de la adolescencia y del inicio de la etapa adulta es el momento para los jóvenes de establecer nuevas metas e incrementar la independencia y la responsabilidad (Lerner, 1995). Para muchos jóvenes, la transición de la adolescencia a la vida adulta marca el comienzo de la independencia económica y la búsqueda y establecimiento de relaciones significativas fuera de las redes familiares. Perseguir estos objetivos a menudo incrementa la distancia entre los jóvenes adultos y sus abuelos, limitando las oportunidades de contacto. Los hallazgos de algunos investigadores al ver que la frecuencia de contacto entre los abuelos y los nietos declina con la edad han llevado a concluir que esta relación intergeneracional tiene una importancia periférica para la mayoría de los jóvenes adultos. Sin embargo, recientemente se han cuestionado estas interpretaciones. Algunos autores (Connidis, 1989) han argumentado que la adultez puede proporcionar una oportunidad para mejorar y estrechar los lazos entre abuelos y nietos. En este sentido, hay ya un cuerpo de investigaciones, aunque escaso todavía, 
sugiriendo que los jóvenes adultos ven las relaciones con sus mayores como muy importantes e influyentes en sus vidas (Brussoni y Boon, 1998).

Para algunos autores, la relación abuelos-nietos no cambia nunca. Para otros, hay una serie de cambios asociados a la edad de los nietos que comienzan en la adolescencia (Tyszkowa, 1993), posiblemente porque hacia el final de esta etapa los nietos tienden a focalizar su energía en el desarrollo personal y en las relaciones fuera de la familia (Roberto y Stroes, 1992). Así, y de modo natural, conforme los nietos van creciendo, las relaciones con los abuelos cambian: de compañeros de juegos; los abuelos pasan a ser confidentes y amigos (Dellman-Jenkins, 1987). Cada vez existe una mayor evidencia que muestra cómo el contacto entre los abuelos y los nietos conforme éstos se van haciendo adultos es no ritualístico u obligatorio, sino voluntario. Autores como Hodgson (1992), Johnson (1983), Kivett (1985), Sprey y Mathews (1982) o Thomas (1986) concluyen en sus estudios transversales que la relación entre abuelos y nietos se debilita conforme aumenta la edad. A la misma conclusión llegan Field y Minkler (1988) en su estudio longitudinal, quienes demostraron que la frecuencia de contacto con los nietos declina sobre los catorce años. A pesar de la menor frecuencia de contacto hallada, Field y Minkler (1988) no encontraron un declive paralelo en el nivel de satisfacción, lo cual sugiere quizás que el aumento de autonomía en la relación entre abuelos y nietos debe ser un cambio normativo y aceptado dentro de esta relación.

Las relaciones abuelos-nietos pueden seguir un patrón cíclico durante el curso de la vida, representables gráficamente por una curva con picos y descensos a lo largo de todo el ciclo vital (Cherlin y Furstenberg, 1986). De manera consistente con las posiciones teóricas en torno a la familia nuclear mantenidas por Hill y Holmbeck (1986), y según el trabajo de Creasey y Koblesky (1991), al final de la adolescencia no se daría una ruptura importante en las relaciones entre abuelos y nietos. La mayor parte de los nietos indicaban que existía amor y respeto mutuo en sus relaciones con los abuelos, así como niveles muy bajos de conflicto. Las nietas indicaban, con más frecuencia que los nietos, relaciones más estrechas (sobre todo en apoyo instrumental, admiración y afecto), coincidiendo con los resultados de Eisenberg (1988). Por otro lado, sin embargo, existe muy poca investigación centrada en la percepción que tienen los nietos del rol que desempeñan los abuelos en sus vidas. Hodgson (1998) resume en cinco las dimensiones que se han tenido en cuenta en los diferentes estudios sobre las relaciones entre los abuelos y los nietos: factores mediadores (linaje y género, proximidad), intercambio de apoyo tanto emocional como instrumental, frecuencia de contacto y significado del rol, afecto y papel que tiene la generación intermedia.

Muchas investigaciones sugieren que las relaciones entre abuelos y nietos a lo largo del ciclo vital se caracterizan más por la continuidad que por el cambio. Informes retrospectivos realizados por nietos adultos sobre el grado en el que su niñez estuvo influida por sus abuelos, predicen la cercanía emocional en sus 
relaciones intergeneracionales contemporáneas (Mathews y Sprey, 1985). Es cierto que en el estudio de Creasey y Kaliher (1994) los nietos más mayores expresaron un menor deseo de contacto, de ayuda instrumental, de satisfacción en la relación, de intimidad, de admiración y de fidelidad que los menores; sin embargo, las diferencias de edad estaban ausentes en lo que hace a la percepción del afecto por los abuelos. Los resultados obtenidos por Creasey y Kaliher (1994) apoyaron, con matices, la hipótesis sobre la menor importancia de los familiares y de otras figuras adultas como agentes proveedores de apoyo durante la transición de la preadolescencia a la adolescencia. Sus datos demostraron que aunque los abuelos ejercen una influencia decreciente como fuente de apoyo, las relaciones interpersonales con sus nietos siguen siendo cualitativamente fuertes, y rara vez los nietos valoran su relación con los abuelos como no óptima, con independencia de su edad. Con respecto a los cambios en la relación abuelos-nietos, existen diversas investigaciones que indican la importancia de la relación del abuelo con su hijo, como predictor de una buena relación entre el abuelo y el nieto (Drew y Smith, 1999). También algunos autores han hablado del papel de los abuelos como elemento de estabilidad en momentos de crisis familiar (Hagestad, 1985; King, 2003).

Con respecto a la variable género, los nietos y las nietas tienen un contacto mayor con las abuelas que con los abuelos, lo que sugiere que el género del abuelo puede ser más importante que la proximidad a la hora de determinar la relación (Silverstein, Giarrusso y Bengtson, 1998). En el caso de los cuidados de los nietos y otras tareas de apoyo, las madres jóvenes aprecian totalmente este apoyo de las mujeres mayores. Entre otras cosas, son las abuelas concretamente la principal estrategia para que las mujeres puedan compatibilizar el trabajo y la familia (Tobío, Fernández-Cordón y Agulló, 1998). Existen también importantes diferencias en lo que hace a la percepción del afecto y la admiración, ya que en general las nietas refieren una mayor percepción de afecto que los nietos y ven a las abuelas más afectuosas que a los abuelos. Aunque nietos y nietas respetan mucho a sus abuelos, los datos revelan que admiran más a las abuelas que a los abuelos (Creasey y Koblesky, 1991). También en la literatura científica encontramos diferencias de género al preguntar a los abuelos y abuelas. Thomas (1986) halló que las abuelas se encuentran más satisfechas con su rol que los abuelos. Esto se debe quizás a su mayor experiencia con las relaciones familiares íntimas, ya que las mujeres suelen ser las principales responsables de la crianza de sus hijos. Los hombres que expresaron mayor satisfacción eran los más mayores, que tenían una relación activa con sus nietos jóvenes y se hallaban felices con su implicación en las tareas de crianza.

Hay dos principales estrategias que utilizan las madres solas para compatibilizar familia y empleo basadas en la sustitución de la madre en su ausencia por otra mujer que asume el rol de ama de casa - según los datos recogidos por Tobío y Fernández-Cordón (1999) - En un caso, esa madre vicaria es un miembro de la familia extensa, generalmente la madre de la mujer trabajadora; en el otro, es una 
RIS

REVISTA INTERNACIONAL DE SOCIOLOGIA

№ 38, MAYO-ACOSTO, 2004

SACRAMENTO PINAZO Y JULIÁN MONTORO

persona que realiza esa actividad a cambio de una remuneración económica. La ayuda de la familia extensa, en especial de la abuela materna, es el recurso principal con el que cuentan las mujeres solas que tienen hijos y trabajan. La relación con la familia del padre de sus hijos suele ser mucho más distante, aunque, en ocasiones, recuperan indirectamente su función de abuelos cuidadores en los periodos de fin de semana o vacaciones, durante los que los niños están con su padre. El papel de la abuela ayudando a su hija y sus nietos es tan importante que llega a ser una condición para la viabilidad de las familias monoparentales en las que la madre trabaja. Se observa una construcción social del rol de cuidadora (rol fuertemente internalizado a través de la socialización), basado en las creencias tradicionales en torno a una "naturalización" y suposición social de la mujer como mejor cuidadora (Bazo y Domínguez-Alcón, 1996).

Creasey y Koblesky (1991) no encontraron diferencias asociadas al linaje. Las nietas no indicaron una relación más positiva con abuelas maternas ni tampoco los nietos expresaron mantener mejores relaciones con abuelos paternos. Los autores, sin embargo, hallaron un efecto significativo del factor género, indicando que las nietas preferían más que los nietos estar con ambos abuelos; que los nietos no discriminaban entre abuelos y abuelas como fuente de compañía; que nietos y nietas adolescentes referían más conflictos con sus abuelos que con sus abuelas, aunque la mayor parte de los nietos y nietas tenían pocos o ningún conflicto con sus abuelos y abuelas. También con respecto a la ayuda instrumental suministrada se perciben diferencias relacionadas con el género: las nietas dan cuenta de más ayuda recibida de sus abuelos y abuelas que los nietos. Diferenciando por dimensiones, en el estudio de Creasey y Koblesky (1991) los autores encontraron que las abuelas reciben valoraciones más altas que los abuelos (en aspectos como intimidad, afecto, admiración, apoyo instrumental, poder y alianza), aunque no se detectaron diferencias significativas por razón de género en lo que hace a la satisfacción en la relación y en el deseo de compañía, lo que contrasta con diversos estudios empíricos que han analizado a los adolescentes tardíos a este respecto (Hartshorne y Manaster, 1982, Hoffman, 1979; Mathews y Sprey, 1985). Una posible explicación para estos hallazgos divergentes radicaría en que en este estudio se tuvo especial cuidado en controlar la influencia tanto de las enfermedades físicas como cognitivas, que tendencialmente afectan más a los abuelos que a las abuelas, dado que los hombres suelen enfermar antes que las mujeres, lo que a su vez está demostrado que afecta decisivamente a las relaciones entre los abuelos y los nietos (Creasey y Jarvis, 1989). También Kennedy (1991) encontró similares resultados: las abuelas son elegidas en el doble de ocasiones que los abuelos y, además, aquellos nietos que eligieron a las abuelas manifestaron tener relaciones más cercanas, sentirse más comprendidos por sus abuelas y comprenderlas mejor que a sus abuelos. Los abuelos maternos (abuelo y abuela) son los que más influyen en las vidas de la mayor parte de nietos y nietas (Hagestad y Speicher, 1981). 
Los abuelos sirven como fuerza estabilizadora y actúan como un recurso para sus hijos y nietos, pudiendo proveer una variedad de servicios concretos a sus nietos, sobre todo, cuando son jóvenes. La mitad de los nietos jóvenes adultos entrevistados refieren retrospectivamente que sus abuelos les proveyeron de apoyo emocional, apoyo instrumental y apoyo financiero durante su infancia (Eisenberg, 1988). A pesar de ello, el balance de apoyo cambia cuando los nietos llegan a la adultez; los abuelos refieren que reciben más apoyo expresivo e instrumental de sus nietos adultos del que ellos dan. Así lo muestra el trabajo de Langer (1990) y el de Kivett (1985). En el estudio de Kivett (1985) sobre una muestra de abuelos encontró que tres cuartas partes de ellos sentían que sus nietos eran responsables de proveerles de apoyo financiero y ayuda instrumental en los momentos de necesidad. En términos de ayuda instrumental las nietas parecen otorgar un mayor apoyo a sus abuelos que los nietos (Creasey y Koblesky, 1991).

La realización de actividades conjuntas es un vehículo para la expresión del afecto y un medio para el conocimiento mutuo, así como cauces de influencia y de asistencia. Para algunos autores como Roberto y Stroes (1992) y Kennedy (1989), la frecuencia con la que nietos y abuelos realizan actividades conjuntas es muy baja. Para los citados autores, los tipos más frecuentes de interacción fueron las visitas breves para conversar acerca de temas importantes. Algunos autores han investigado las actividades realizadas conjuntamente por abuelos y nietos preguntando o bien a los nietos (Kornhaber y Woodward, 1981; Kennedy, 1992b), o bien a los abuelos (Robertson, 1976; Kivnick, 1982; Cherlin y Furstenberg, 1986; Baranowski, 1982). En el estudio de Kennedy (1992b) se interrogó a 391 nietos jóvenes adultos estudiantes universitarios acerca de la importancia que una serie de 29 actividades (generada a partir de una escala abierta formulada por 273 nietos jóvenes adultos) tenían en la relación con sus abuelos, proporcionando sus respuestas un ranking de la prevalencia de cada actividad cuando abuelos y nietos pasaban tiempo juntos. Estas 29 actividades constituyen un listado operativo y diverso que ha sido agrupado en seis categorías: sociabilidad (hablar de temas importantes para ambos, ver la televisión), compañerismo (pasear, leer), ayuda doméstica (cocinar, comprar), acontecimientos comunitarios (deportes, fiestas), entretenimiento (salir a comer a un restaurante, ir de compras), ayuda en tareas al aire libre (jardinería, gestiones administrativas). Concluye el autor que a mayor cercanía en la relación, más diversas son las actividades que realizan conjuntamente y, recíprocamente, cuantas más actividades se comparten, mayor es la sensación de cercanía. Las nietas compartirían más actividades conjuntas que los nietos, realizándolas más con las abuelas que con los abuelos.

Tyszkowa (1991), en un estudio realizado en Polonia, encontró que las actividades realizadas más frecuentemente entre abuelos y nietos (adolescentes y jóvenes) de manera conjunta eran: conversaciones (52\%), paseos (24\%) y juegos (17\%). Las conversaciones mantenidas por los nietos adolescentes y adultos con sus abuelos parecen ser de particular significación para los nietos, porque los 
padres trabajadores no tienen el tiempo o la paciencia suficiente para conversar con sus hijos, y porque la existencia al mismo tiempo de un vínculo mutuo y de una cierta distancia respecto de sus abuelos y abuelas les permite hablar de asuntos que no abordarían con sus padres. Otras actividades llevadas a cabo conjuntamente con los abuelos y abuelas hacen referencia al ocio, al aprendizaje de habilidades prácticas y a la iniciación en las tradiciones familiares (Hyde y Gibbs, 1993). La investigación sobre las relaciones intergeneracionales muestran cómo, de entre las actividades que realizan abuelos y nietos hasta los 13 años, las vinculadas al cuidado son las que descienden en la adolescencia, y, por el contrario, se mantienen la conversación, la ayuda económica, la comunicación telefónica o el paseo (CIREM, 1994). Un papel fundamental de la familia es la transmisión de valores de una generación a otra. Los abuelos pueden jugar un rol crucial en este proceso, sirviendo de árbitros entre padres e hijos con respecto a los valores centrales para la continuidad familiar y el desarrollo individual (Pinazo, 1999a, 1999b).

La escasez de trabajos en lengua castellana y en particular en España sobre las relaciones abuelos-nietos nos llevó a iniciar en el año 1994 una línea de investigación sobre este tema de creciente interés, fruto de la cual es el trabajo que aquí presentamos. Además de tomar la frecuencia de contacto o el número de actividades compartidas como indice de la calidad de la relación intergeneracional (como hicieron Kornhaber y Woodward, 1981 o Kennedy, 1992b), la presente investigación examina diversos aspectos que pueden influir en la calidad del vínculo intergeneracional, preguntando sobre la cercanía o intimidad, nivel de conocimiento e influencia mutua entre abuelos nietos. Nuestro objetivo de investigación ha sido examinar los factores determinantes de la relación intergeneracional entre los abuelos y los nietos e identificar la influencia de las variables independientes sobre la calidad de la relación intergeneracional entre nietos y abuelos. Finalmente, examinamos la relativa importancia de cada uno de los grupos de variables utilizadas en el modelo.

\section{METODOLOGÍA}

\section{Muestra y procedimiento de muestreo}

El estudio incluye una muestra de 361 adolescentes y jóvenes de entre 15 y 25 años en la Comunidad Valenciana. Durante la primavera del año 1999 se contactó con estudiantes de Ciencias Sociales de la Universidad de Valencia para que distribuyeran y recogieran cuestionarios rellenados por adolescentes y jóvenes de su entorno relacional. Los entrevistadores recibieron a lo largo de tres sesiones entrenamiento para la selección de los participantes, la administración del cuestionario y el objetivo de la investigación. Cada entrevistador fue instruido para entrevistar a cuatro adolescentes y jóvenes incluyendo una de cada una de las 
siguientes condiciones: estudiante en centro privado de bachillerato, estudiante en centro público de bachillerato, estudiante no universitario y, finalmente, estudiante de la Universidad. Un total de 450 cuestionarios fueron distribuidos. Los entrevistadores indicaron que la mayor parte de los contactados decidieron responder a las preguntas. Igualmente pocos cuestionarios fueron rechazados por su falta de datos. Las características de la muestra final se pueden apreciar con mayor detalle en la Tabla 1. La mitad de los participantes son varones y en un porcentaje bastante alto (84\%) provienen de familias identificadas como intactas.

\section{El cuestionario y su análisis}

El examen de los factores que influyen en las relaciones intergeneracionales entre abuelos-nietos se realiza con el empleo de un cuestionario, construido especialmente para esta investigación, pero que incluye diversos instrumentos y variables utilizadas en investigaciones previas. Todas las preguntas sobre la relación de los adolescentes y jóvenes con los abuelos se refieren a su relación con el abuelo/a preferido/a. Las variables independientes consideradas en el análisis incluyen:

\section{a) Aspectos demográficos}

Género (varón=1; mujer=0); edad cronológica $(15-17=1,18-21=2,22-25=3)$; tipo de familia (intacta $=1$; otra $=0$ ); número de nietos; número de abuelos vivos; abuelo preferido, que se compone de cuatro variables que indican preferencia para el abuelo/a materno/a (tipo abuelo $=1$; otro abuelo $=0$ ); proximidad-residencia (barrio=4, ciudad $=3,<10 \mathrm{~km}=2,10-40 \mathrm{~km}=1,>40 \mathrm{~km}=0$ ); frecuencia de la relación (diariamente $=4$, semanal $=3$, mensual $=2$, semestral $=1$, anual $=0$ ); y salud del abuelo/a (buena $=2$, regular $=1$, mala $=0$ ).

\section{b) La percepción de la calidad de la relación entre abuelos y padres}

Esta percepción es la que tienen los entrevistados, medida con un índice de dos indicadores sobre la cercanía o intimidad de la relación entre ambos (nada=1, poco $=2$, mucho $=3$ ), y la frecuencia de contacto entre abuelos y padres (nada $=1$, poco $=2$, frecuente $=3$ ). El índice compuesto con los dos indicadores (suma de los valores) revela que padres y abuelos mantienen un contacto e intimidad frecuente y elevado (Media= 5.4, $\mathrm{SD}=0.8$ ) (Tabla 1 ). La consistencia interna o fiabilidad para este índice es aceptable (Cronbach alpha $=0.73$ ).

\section{c) El tipo de actividades intergeneracionales}

El concepto de "actividades intergeneracionales" se ha referido en el cuestionario a las actividades que los nietos realizan con sus abuelos/as favoritos. Basándonos 
RIS

REVISTA INTERNACIONAL DE SOCIOLOCIA

№ 38, MAY0-ACOST0, 2004

SACRAMENTO PINAZO Y JULLÁN MONTORO

en el estudio de Kennedy (1992b) y en la investigación cualitativa realizada por Pinazo (2002), se seleccionaron 18 posibles actividades, que fueron agrupadas en tres dimensiones sobre la base de los resultados del análisis factorial: a) actividades de acompañamiento (conversar, pasear, visitar a familiares y amigos, discutir y tomar decisiones importantes, rezar); b) actividades en tareas domésticas (cocinar, limpiar, realizar labores domésticas e ir de compras); y c) actividades de entretenimiento (jugar, hacer excursiones, viajes, preparar y participar en fiestas, escuchar música y bailar). Los resultados del análisis factorial basado en la extracción de Componentes Principales con una rotación Varimax mostró que los catorce indicadores explican casi el $50 \%$ de la varianza total (actividades tareas domésticas 20,9\%; acompañamiento $18,3 \%$; y recreativas $12,2 \%$ ). Cada indicador utiliza una escala Likert de cuatro valores (nunca $=1$, ocasionalmente $=2$, bastante a menudo $=3$ y muy a menudo $=4$ ). Los tres índices se generaron con la suma de los valores de cada indicador mencionado anteriormente, presentando niveles aceptables de consistencia interna (acompañamiento, alpha $=0.75$; ayuda doméstica, alpha $=0.74, \mathrm{y}$ actividades recreativas, alpha $=0.68$ ).

\section{d) El rol desempeñado por los abuelos favoritos con sus nietos}

Se examina preguntando a los nietos participantes en el estudio sobre seis tipos diferentes de roles aplicados frecuentemente a los abuelos/as: cuidador (escucha, cuida a los nietos), indulgente (consiente a los nietos), promotor de la entidad familiar y la continuidad (completa detalles de la niñez de los padres y otras historias familiares), maestro (imparte enseñanzas de destreza y conocimientos), modelo de rol-valores tradicionales (insiste en valores tradicionales) y transmisor de valores (actividades religiosas, dador de consejos). El rol se mide a partir de la frecuencia con que los abuelos/as ejercen cada uno de estos roles, y se utiliza una escala de intervalo con tres valores (nunca $=0$, pocas veces $=1$, muchas $=2$ ). Las variables ordinales que se refieren a cada uno de los roles presentan una distribución normal y niveles aceptables de asimetría y curtosis.

Finalmente, la variable dependiente, la percepción de la calidad de la relación intergeneracional abuelos-nietos se obtuvo preguntando a los jóvenes sobre: a) el grado de cercanía con sus abuelos; b) el nivel del conocimiento del abuelo con respecto a nietos y viceversa; c) el grado de influencia del abuelo/a en las creencias de los nietos y d) el grado de satisfacción con los abuelos y de éstos con los nietos. Cada uno de los indicadores se mide con una escala de tres valores (nada $=1$, poco $=2$, mucho $=3$ ) y el nivel de fiabilidad o consistencia interna para el índice compuesto de los seis indicadores (suma de sus valores) es muy aceptable (Cronbach alpha $=0.81$ ). En conjunto, los participantes del estudio consideran que el vínculo o relación con los abuelos preferidos es más bien moderado (Media=13.8; $\mathrm{SD}=2.6$ ) (ver Tabla 1). 
Tabla 1

Características de la muestra $(N=361)$

\begin{tabular}{|c|c|c|}
\hline Variables Independientes & $\mathrm{N}$ & $\%$ \\
\hline \multicolumn{3}{|l|}{ Edad } \\
\hline $15-17$ & 71 & 19.7 \\
\hline $18-21$ & 143 & 39.6 \\
\hline $22-25$ & 147 & 40.7 \\
\hline \multicolumn{3}{|l|}{ Género } \\
\hline Varón & 174 & 49.2 \\
\hline \multicolumn{3}{|l|}{ Residencia } \\
\hline Medio Urbano & 305 & 85.0 \\
\hline Co-Residencia & & \\
\hline El abuelo/a vive solo & 49 & 13.6 \\
\hline \multicolumn{3}{|l|}{ Estructura Familia } \\
\hline Familia Intacta & 304 & 84.2 \\
\hline \multicolumn{3}{|l|}{ Número de Nietos } \\
\hline Media (SD) & 2.7 & $(0.7)$ \\
\hline \multicolumn{3}{|l|}{ Número de Abuelos Vivos } \\
\hline Media (SD & 2.1 & $(0.9)$ \\
\hline Preferencia de Abuelo/a & $\mathrm{N}$ & $\%$ \\
\hline Abuela Materna & 198 & 55.2 \\
\hline Abuela Paterna & 71 & 19.8 \\
\hline Abuelo Materno & 49 & 13.6 \\
\hline Abuelo Paterno & 41 & 11.4 \\
\hline \multicolumn{3}{|l|}{ Proximidad-Residencia } \\
\hline Mismo barrio & 170 & 47.4 \\
\hline Misma ciudad & 91 & 25.3 \\
\hline$<10 \mathrm{KM}$ & 24 & 6.7 \\
\hline $10-40 \mathrm{KM}$ & 22 & 6.1 \\
\hline$>40 \mathrm{KM}$ & 52 & 14.4 \\
\hline \multicolumn{3}{|l|}{ Frecuencia de Relación } \\
\hline Diariamente & 128 & 35.8 \\
\hline 1 vez por semana & 130 & 36.3 \\
\hline 1 vez por mes & 54 & 15.1 \\
\hline Cada 1-6meses & 32 & 8.9 \\
\hline 1 vez por año & 14 & 3.9 \\
\hline \multicolumn{3}{|l|}{ Salud Abuelo/a Favorito } \\
\hline Buena & 193 & 53.5 \\
\hline Regular & 138 & 38.2 \\
\hline Mala & 30 & 3.9 \\
\hline \multicolumn{3}{|l|}{ Relación Padre/Abuelos/as } \\
\hline Mínimo-Máximo & 2 & 6 \\
\hline Media (SD) & 5.4 & $(0.8)$ \\
\hline
\end{tabular}


Tabla 1

Caracteristicas de la muestra (continuación) $(N=361)$

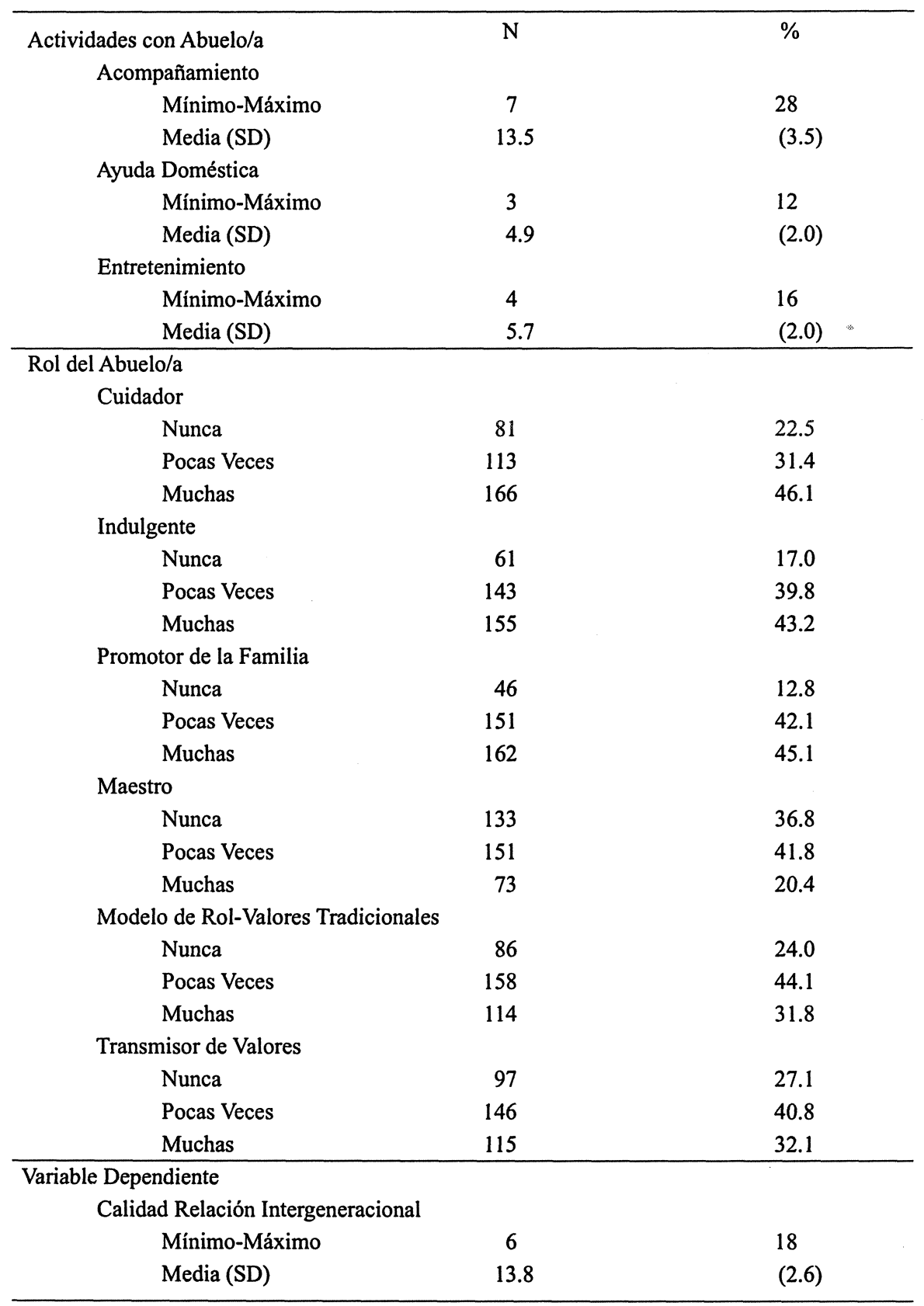

158 


\section{RESULTADOS}

El análisis de los factores que influyen en la calidad de las relaciones intergeneracionales entre abuelos y los nietos se realiza con la técnica multivariable de la regresión. El modelo a verificar incluye las variables sociodemográficas, el contacto o relación entre padres y abuelos, las actividades intergeneracionales y el tipo de rol atribuido a los abuelos. Dos son los temas de investigación que suscitaron nuestro interés: en primer lugar, identificar la influencia de las variables independientes sobre la calidad de la relación intergeneracional entre nietos y abuelos; y en segundo lugar, determinar la relativa importancia de cada uno de los grupos de variables utilizadas en el modelo. La Tabla 2 presenta los resultados de la regresión múltiple. Se incluyen los datos de cada variable (coeficientes no estandarizados y los beta o estandarizados) y del conjunto. El método de regresión utilizado es el de la entrada simultánea de las variables. Cada uno de los modelos (I-IV) corresponde al análisis realizado con la adición de variables independientes.

Los resultados finales (ver Modelo IV) indican que las variables independientes explican en conjunto un elevado porcentaje de la variable dependiente (R-squared $=0.48$ ), lo que significa que los factores sociodemográficos, la relación de los padres con los abuelos, el tipo de actividades intergeneracionales y el tipo de roles ejercidos por los abuelos son determinantes de casi la mitad de la variabilidad en la calidad del vínculo intergeneracional. De forma específica, los nietos del estudio indican que dicha relación está determinada de forma positiva predominantemente por el grado de contacto o intimidad entre los padres y los abuelos $(B e t a=0.30)$, sugiriendo que, a mayor contacto entre padres y abuelos, mayor calidad en la relación intergeneracional entre nietos y abuelos.

Otros factores significativos que contribuyen a la mejora de la relación intergeneracional son los tres siguientes:

a) La existencia de actividades de acompañamiento y de apoyo emocional entre abuelos y nietos $(\mathrm{Beta}=0.21)$. La calidad del vínculo entre abuelos/nietos se beneficia, pues, de una mayor frecuencia de actividades, tales como conversar, pasear, mirar recuerdos familiares, visitar familiares, discutir y tomar decisiones, y rezar.

b) La frecuencia de relación entre nietos y abuelos/as. Este aspecto significativo sugiere que la relación intergeneracional mejora con una mayor frecuencia de contacto de los nietos con los abuelos/as preferidos (Beta $=.13 ; \mathrm{p}<0.05$ ).

c) Dos de los estilos de ejercer la posición de abuelo están igualmente asociados con la mejora en la relación intergeneracional, en particular, la percepción del abuelo ejerciendo el rol de cuidador $(\mathrm{Beta}=.10 ; \mathrm{p}<0.05), \mathrm{y} / \mathrm{o}$ rol de maestro $(\mathrm{Beta}=0.11 ; \mathrm{p}<0.05)$.

d) Otro aspecto que, aunque no llega a ser significativo parece mostrar cierta influencia, sería la tendencia de los nietos a mantener o desarrollar una relación más cercana e intima con la abuela materna que con el resto de los otros abuelos $($ Beta $=0.07 ; \mathrm{p}<0.10)$. 
RIS

REVISTA INTERNACIONAL DE SOCIOLOCIA

№ 38, MAYO-ACOSTO, 2004

SACRAMENTO PINAZO Y JULLÁN MONTORO

Tabla 2

Regresión Múltiple

Factores Determinantes la Relación Intergeneracional Abuelos y Nietos

\begin{tabular}{|c|c|c|c|c|}
\hline & Modelo I & Modelo II & Modelo III & Modelo IV \\
\hline \multicolumn{5}{|l|}{ Variables Independientes } \\
\hline \multicolumn{5}{|l|}{ Sociodemográficas: } \\
\hline Edad & $-.29(-.08) \#$ & $-.28(-.08) \#$ & & \\
\hline \multicolumn{5}{|l|}{ Género } \\
\hline \multicolumn{5}{|l|}{ Medio Urbano } \\
\hline \multicolumn{5}{|l|}{ Vive Solo } \\
\hline \multicolumn{5}{|l|}{ Familia Intacta } \\
\hline \multicolumn{5}{|l|}{ Número de Nietos } \\
\hline Número de Abuelos Vivos & $.24(.09) \#$ & $.25(.09)$ & & \\
\hline Preferencia Abuela Materna & $.53(.10)$ & $.45(.08) \#$ & $.40(.07) \#$ & \\
\hline Proximidad/Residencia & $.23(.13)$ & $.23(.13)$ & & \\
\hline Frecuencia de Relación & $1.1(.47)$ & $.62(.26)$ & $.34(.14)$ & $.32(.13)$ \\
\hline \multicolumn{5}{|l|}{ Salud Abuelo Favorito } \\
\hline Relación Padre/Abuelos & & $1.2(.41)$ & $1.0(.34)$ & $.89(.30)$ \\
\hline \multicolumn{5}{|l|}{ Actividades con Abuelo } \\
\hline Acompañamiento & & & $.22(.30)$ & $.15(.21)$ \\
\hline Ayuda Doméstica & & & $.13(.10)$ & \\
\hline \multicolumn{5}{|l|}{ Entretenimiento } \\
\hline \multicolumn{5}{|l|}{ Rol del Abuelo } \\
\hline Cuidador & & & & $.34(.10)$ \\
\hline \multicolumn{5}{|l|}{ Indulgente } \\
\hline \multicolumn{5}{|l|}{ Promotor de la Familia } \\
\hline Maestro & & & & $.38(.11)$ \\
\hline \multicolumn{5}{|l|}{ Tradicional/Transmisor Valores } \\
\hline Adjusted R-Squared & .22 & .34 & .45 & .48 \\
\hline R-Squared Change & & .10 & .11 & .04 \\
\hline F-Test & 9.7 & 14.8 & 18.9 & 16.3 \\
\hline $\mathrm{N}=361(\# \mathrm{p}<.10)(\mathrm{p}<.05)$. & & & & \\
\hline
\end{tabular}

*Coeficientes no estandarizados. Coeficientes estandarizados en paréntesis

160 
En lo referente a la relativa importancia de los diversos factores, el análisis de la regresión múltiple por etapas o jerarquizado (ver Modelos I-IV) indica que cada uno de los diferentes grupos de variables contribuye de forma significativa (ver $r$-squared change para cada modelo) a la explicación de la calidad de la relación intergeneracional o variable dependiente. En el Modelo I, en el que tan sólo las variables sociodemográficas se toman como factores de predicción, la explicación total de la variable dependiente es moderada $(R$-squared $=.22)$, siendo la frecuencia de contacto con los abuelos, la proximidad y la preferencia por la abuela materna, las variables significativas. La adición de la variable sobre calidad de relación entre padres y abuelos (ver Modelo II) supone un incremento del 10\% sobre el modelo anterior. Igualmente, la introducción de las variables sobre las actividades compartidas con los abuelos supone otra mejora similar (ver Modelo III), produciendo un resultado con menor peso específico para los factores sociodemográficos. Finalmente, la inclusión de las variables de los roles atribuidos a los abuelos produce también un incremento significativo, aunque menor que en la predicción de la variable dependiente (ver Modelo IV).

\section{CONCLUSIONES}

El análisis de los datos identifica aspectos sociodemográficos y actitudinales que son determinantes de la calidad de las relaciones intergeneracionales entre los abuelos y los nietos para la muestra de jóvenes de la Comunidad Valenciana. Los datos del análisis en su conjunto confirman los resultados de los estudios previos fundamentalmente en la literatura anglosajona: en particular, la importancia que la relación entre padres y abuelos tiene en el mantenimiento de relaciones positivas intergeneracionales entre los nietos y los abuelos, así como la asociación positiva con la frecuencia de contacto y la realización de actividades de acompañamiento (conversar, pasear, visitar a familiares y amigos, discutir y tomar decisiones importantes, rezar). A continuación se hace un comentario de los aspectos descriptivos más relevantes de dicha relación intergeneracional, así como de los resultados del análisis multivariable sobre los factores que determinan la calidad de la relación intergeneracional nietos-abuelos, y de la importancia relativa de cada uno de los mismos.

Los datos descriptivos indican que, en su mayoría, los nietos identifican como favorita la figura de la abuela, siendo la abuela materna la citada en primer lugar (55\%), seguida de la paterna (20\%) (Tabla 1). Nuestros resultados a este respecto coinciden con los de Hartshorne y Manaster (1982), quien encontró que, cuanto mayor era el contacto con los abuelos, más satisfechos estaban los nietos, y que los nietos tenían más contacto con su abuela materna. Al preguntar acerca de la importancia de cada abuela individualmente, la abuela materna recibía las cotas más altas. Muchos estudios apoyan la idea de que los abuelos maternos son los 
más influyentes en las vidas de los nietos (Hoffman, 1979; Hagestad y Speicher, 1981; Eisenberg, 1988). Con respecto al desarrollo de valores, Roberto y Stroes (1992) indicaron que nietos y nietas perciben que las abuelas son más influyentes en sus vidas que los abuelos. En cuanto a actividades compartidas, los nietos participan más en actividades con sus abuelas que con sus abuelos. Roberto y Stroes (1992) encontraron que los abuelos maternos eran elegidos en primer lugar y, en particular, las abuelas. Incluso para la realización de actividades, los nietos y las nietas participan más en actividades con sus abuelas, sobre todo en conversaciones acerca de cosas importantes en su vida diaria, ayuda en tareas domésticas y visitas breves.

Respecto de la frecuencia de contacto y tipo de relación, los abuelos preferidos residen relativamente cerca de sus nietos, bien en el mismo barrio (47\%) o bien en la ciudad (25\%). En su mayoría, los abuelos comparten residencia con otros familiares, siendo muy pocos los abuelos que viven solos (14\%) y su salud es percibida como buena y regular (82\%). Los nietos reconocen mantener con sus abuelos una frecuencia de relación de tipo semanal (36\%) y diaria (36\%). Los tipos de interacción más frecuentes con los abuelos son las visitas breves para conversar y la participación en discusiones importantes. Los abuelos son vistos por sus nietos como confidentes y compañeros. Comparando con la literatura existente, también Hyde y Gibbs (1993), Tyszkowa (1991) y Eisenberg (1988) encontraron que las actividades más frecuentes que realizan los nietos con sus abuelos son conversar y escuchar, sobre todo con las abuelas maternas y en actividades que favorecen la cercanía emocional. En un estudio realizado en la Comunidad Valenciana con abuelas, Benlloch y Berjano (1994) hallaron que cuanto mayor era la frecuencia de contactos entre la abuela y el nieto, mayor era la satisfacción de ésta. Resultados éstos congruentes con la investigación de Eisenberg (1988). Los nietos indican igualmente (87\%) que los abuelos realizan en su mayoría actividades relacionadas con su papel de promotores de la familia y la continuidad familiar. El predominio de este papel de los abuelos es consistente con otros estudios que han examinado los diferentes modelos de rol para los mayores. Por ejemplo, los nietos en el estudio de Roberto y Stroes (1992) percibieron que sus abuelos (sobre todo sus abuelas) eran muy influyentes en el desarrollo de sus valores (ideales familiares, identidad personal y creencias religiosas).

Los resultados del análisis multivariable de la muestra de nietos confirman varias de las conclusiones que han sido alcanzadas en estudios previos (Hagestad, 1985; Eisenberg, 1988). En primer lugar, los datos muestran que los nietos parecen otorgar una influencia mayor a las abuelas maternas en comparación a la abuela paterna y a ambos abuelos. Esta preferencia parece corroborarse con los datos sobre actividades que los nietos desarrollan con sus abuelas (Roberto y Stroes, 1992). En la literatura anglosajona merece destacarse el trabajo de Robertson (1976), quien entrevistó a nietos y nietas de 18 a 26 años, hallando que los nietos y las nietas se encontraban a gusto con sus abuelos, tenían actitudes favorables hacia 
ellos, a la vez que consideraban a sus abuelos una fuente de influencia y se sentían responsables de prestarles apoyo y ayuda. También Kennedy (1992a) encontró que la proximidad, la buena relación entre abuelos y padres, junto a la creencia de ser conocido y conocer al abuelo, se asociaban a una buena relación entre los nietos jóvenes adultos y sus abuelos. En la muestra española destaca el trabajo realizado con adolescentes por Triadó, Martínez y Villar (1999), cuyos resultados mostraron que los nietos perciben a los abuelos maternos como más cercanos y con roles más positivos, mientras que los paternos juegan roles más distantes. Cuando preguntaron a abuelos y abuelas españoles (Triadó y Villar, 2000) sobre la percepción de la relación con sus nietos, los abuelos más jóvenes tendían a mantener mayor cercanía emocional con sus nietos y el balance de intercambios y ayuda entre abuelos y nietos tendía a cambiar con la edad. A medida que los abuelos son más mayores, mencionan menos frecuentemente la prestación de ayuda (económica o cuidados) y más la demanda y recepción de ayuda por parte de sus nietos.

Sería interesante, en posteriores investigaciones, analizar díadas de abuelos con sus respectivos nietos, para poder explorar las diferentes percepciones con un grado mayor de exactitud (Triadó et al., 2004). También, análisis longitudinales podrían explorar cómo los cambios en las actitudes de los padres, en las variables personales, en las variables situacionales y en las circunstancias vitales pueden influir en la relación que los nietos tienen con sus abuelos. Todavía no ha sido estudiada la influencia que la relación abuelos-nietos ocasiona a largo plazo en el bienestar de ambos. Una limitación con la que se han encontrado los trabajos acerca de la relación intergeneracional es que han sido estudios transversales y no longitudinales. Sería necesario para investigaciones posteriores realizar estudios en los que se pudiese analizar el cambio en la relación entre los abuelos y los nietos a través del tiempo. Estos estudios nos ayudarían a indagar de forma más precisa sobre las diferencias de comportamiento de los nietos en su relación con los abuelos, diferencias que pueden ser en ambas direcciones: de los nietos a los abuelos (en cuanto preferencias, actividades y ayuda personal), y de los abuelos hacia los nietos (en cuanto transmisores de valores, tutores o cuidadores). La relación entre la edad de los nietos y su cercanía o vínculo con los abuelos es otro aspecto que esta investigación no ha podido examinar con precisión. El análisis inicial mostraba la existencia de una tendencia negativa (no significativa, p. $<0.10$ ), sin embargo, esta asociación desaparece cuando se controla por aspectos de la relación entre padres y nietos, así como por las diversas actividades y roles de los abuelos con los nietos. Es muy probable que la utilización de grupos de edad para la medición de esta variable sea un factor crítico, en el sentido de que la reducción de la variabilidad que produce dicha operacionalización no haya facilitado el descubrimiento de los efectos reales de la edad de los nietos sobre la relación intergeneracional.

Otro resultado que se desprende del análisis es la importancia del tipo de relación que existe entre padres y abuelos, en el sentido de que a un mayor nivel de 
R I S

REVISTA INTERNACIONAL DE SOCIOLOCIA

№ 38, MAY0-ACOST0, 2004

SACRAMENTO PINAZO Y JULLÁN MONTORO

comunicación, cercanía y contacto entre abuelos y padres corresponde una mejora en la percepción de la calidad de la relación intergeneracional entre los abuelos y los nietos. Este dato es coincidente con los resultados obtenidos por Mathews y Sprey (1985), quienes concluyeron que la cantidad de contacto con los abuelos que los padres faciliten a sus hijos, influirá en el tipo de relación que desarrollarán posteriormente. La naturaleza de la relación abuelo-hijo (padre) es, pues, otro factor importante en el rol de abuelo y su implicación en las vidas de los nietos. Si la relación abuelo-hijo (padre) es armoniosa, esto abre oportunidades para los beneficios directos e indirectos que los abuelos pueden ofrecer a sus nietos y para que los abuelos se sientan cómodos en su rol de apoyo. Si la relación abuelo-hijo (padre) se rompe o aparecen dificultades, esto puede modificar la proximidad de los abuelos a los nietos, el contacto, la implicación y el sentimiento de ostentar un rol satisfactorio (Drew y Smith, 1999). En algunos estudios como el de Creasey y Kaliher (1994) se intentó controlar el estatus marital de la familia nuclear del nieto incluyendo sólo a aquéllos cuyos padres no hubiesen experimentado un divorcio, lo que constituye un acontecimiento vital que pone a prueba los vínculos familiares. La literatura ha puesto de relieve el papel de los abuelos como elemento de estabilidad en momentos de tensión o crisis familiar (Hagestad, 1985). En el trabajo de Kennedy (1992b) los estudiantes de las familias rotas y de las reconstruidas presentan tasas de actividad significativamente altas reflejando quizás las muchas ocasiones que los nietos pasan junto a sus abuelos. Este dato podría ser de interés a la hora de entender la gran importancia que los nietos procedentes de familias monoparentales y reconstruidas dan a las actividades que comparten con sus abuelos. Y esto a su vez sería igualmente de interés a la hora de considerar el derecho de visita de los abuelos y los intereses de los padres no custodios.

En conclusión, por ahora sabemos poco acerca de las relaciones entre los abuelos y los nietos. Este estudio ha intentado, utilizando una muestra no aleatoria de jóvenes, acercarse al análisis examinando aspectos de la calidad en la relación intergeneracional, centrándose en la perspectiva de los nietos jóvenes adultos. Cabe resaltar que, al igual que en los estudios anglosajones, la percepción de los nietos sobre la calidad de la relación entre sus propios padres y abuelos resulta ser el aspecto que mejor predice la relación intergeneracional abuelos-nietos. De los factores sociodemográficos, tan sólo la frecuencia de relación parece ser relevante para esta muestra de jóvenes. Las actividades compartidas, en particular de acompañamiento, son igualmente significativas, así como la percepción del abuelo como proveedor de cuidado y transmisor de enseñanzas de destrezas y conocimientos. Todos los diversos grupos de variables incluidos en el modelo contribuyen en parte a la explicación de la varianza total, llegando hasta un porcentaje relativamente elevado (48\%) en comparación con los estudios previos. Sin embargo, todavía queda algo más de la mitad de la variabilidad en la calidad de las relaciones abuelos-nietos que ha de ser explicada con la aportación de otros factores y variables que por el momento aún no han sido examinados. Es necesario 
realizar futuras investigaciones que continúen explorando las similitudes y las diferencias en las relaciones entre abuelos y nietos. Los resultados de este estudio confirman la presencia de los abuelos en las vidas de los nietos jóvenes adultos. El examen de las respuestas de los nietos nos da una primera idea o aproximación a las variaciones en el tipo y grado de implicación entre nietos y abuelos.

\section{REFERENCIAS BIBLIOGRÁFICAS}

BARANOWSKI, M.D. (1982), "Grandparent-adolescent relations: beyond the nuclear family", Adolescence, $\mathrm{n}^{\circ} 17, \mathrm{pp} .575-584$.

BAZO, M.T. y N. C. DOMÍNGUEZ-ALCÓN (1996), "Los cuidados familiares de salud en las personas ancianas y las políticas sociales", REIS, $\mathrm{n}^{\circ} 73$.

BENLLOCH, V. y E. BERJANO (1994), "Estudio de las relaciones intergeneracionales entre abuelas y nietos", Geriatrika, Vol. 11, 4, pp. 46-51.

BENLLOCH, V., G. CALATAYUD, D. LLOPIS, S. PINAZO y E. BERJANO (1994), "Relaciones intergeneracionales abuela-nietos", IV Jornadas de Intervención Social del COP, Madrid.

BRUSSONI, M.J. y S. D. BOON (1998), "Grandparental impact in young adults' relationships with their closest grandparents: the role of relationship strength and emotional closeness", International Journal of Aging and Human Development, vol. 46, 4, pp. 267-286.

CIREM (1994), Relacions intergeneracionals.Gent gran $i$ adolescents. Estudi d'una relació. Barcelona, Fundació La Caixa.

CONNIDIS, I.A. (1989), Family ties and aging, Toronto, Butterwoths.

CREASEY, G.L. y P. A. JARVIS (1989), "Grandparents with Alzheimer's disease: effects of parental burden on grandchildren", Family Therapy, $\mathrm{n}^{\circ}$ 16, 79-85.

CREASEY, G.L. y P. J. KOBLESKY (1991), “Adolescent grandchildren's relationships with maternal and paternal grandmothers and grandfathers", Journal of Adolescence, 1991, $\mathrm{n}^{\circ} 14$, pp. 373-387.

CREASEY, G.L. y G. KALIHER (1994), “Age differences in grandchildren's perceptions of relations with grandparents", Journal of Adolescence, $\mathrm{n}^{\circ}$ 17, pp. 411-426.

CHERLIN, A. y F. FURSTENBERG (1986), "Grandparents and family crisis", Generations, vol. 10, pp. 26-28.

DELLMAN-JENKINS, M. (1987), "Teenagers' reported interaction with grandparents. Exploring the extent of alienation", Lifestyles, $\mathrm{n}^{\circ} 8$, pp. 35-36.

DREW, L.A. y P. K. SMITH (1999), "The impact of parental separation-divorce on grandparentgrandchildren relationships", International Journal of Aging and Human Development, vol. 48, (3), pp. 191-216 
EISENBERG, A.R. (1988), "Grandchildren's perspectives on relationships with grandparents: the influence of gender across generations", Sex Roles, $n^{\circ} 19$, pp. 205-217.

FIELD, D. y M. MINKLER (1988), "Continuity and change in social support between young-old and old-old or very old age”, Journal of Gerontology, Psychological Sciences, $\mathrm{n}^{\circ} 43$, pp. 100-106.

HAGESTAD, G. (1985), "Continuity and connectedness", en V. Bengtson y J. Robertson, Grandparenthood, Beverly Hills, SAGE.

HAGESTAD, G.O. y J. L. SPEICHER (1981), "Grandparents and family influence: views of three generations". Artículo presentado en el Meeting of the Society for Research in Child Development, Boston.

HARTSHORNE,T.S. y G. J. MANASTER (1982), "The relationship with grandparents: contact, importance, role conception", International Journal of Aging and Human Development, $\mathrm{n}^{\circ} 15$, pp. 233-245.

HILL, J.P. y G. HOLMBECK (1986), “Attachment and autonomy during adolescence”, en G. Whitehurst (ed.), Annals of Child Development, Greenwich, JAI Press.

HODGSON, L.G. (1992), "Adult grandchildren and their grandparents: The enduring bond", International Journal of Aging and Human Development, $\mathrm{n}^{\circ} 34$ (3), pp. 209-225.

(1998), "Grandparents an older grandchildren", en M.E. Szinovacs (ed.), Handbook on Grandparenthood, Westport, Conn., Greenwood Press.

HOFFMAN, E. (1979), "Young adults' relations with their grandparents: an exploratory study", International Journal of Aging and Human Development, $\mathrm{n}^{\circ}$ 10, pp. 299-309.

HYDE, V. y I. GIBBS (1993), “A very special relationship: granddaughters' perceptions of grandmothers", Ageing and Society, n 13, pp. 83-96.

IMSERSO (1995), Las personas mayores en España. Perfiles. Reciprocidad familiar, Madrid, IMSERSO.

JOHNSON, C. (1983), "A cultural analysis of the grandmother", Research on Aging, $\mathrm{n}^{\circ}$ 5, pp. 547-567.

KENNEDY, G.E. (1989), “College students' relationship with grandparents”, Psychological Reports, $\mathrm{n}^{\circ} 64$, pp. $477-478$.

(1991), Developmental influence of grandparents, Paper presented at the Annual Meeting of the National Council on Family Relations, Denver, CO.

(1992a), "Quality in Grandparent/Grandchild Relationships", International Journal of Aging and Human Development, $\mathrm{n}^{\circ} 35$ (2), pp. 83-98.

(1992b), "Shared activities of grandparents and grandchildren", Psychological Reports, $\mathrm{n}^{\circ} 70$, pp. 211-227. 
KING, V. (2003), “The legacy of a grandparent's divorce: consequences for ties between grandparents and grandchildrem", Journal of Marriage and the Family, n 65, pp. 170-183.

KIVETT, V. (1985), "Grandfathers and grandchildren: patterns of association, helping and psychological closeness", Family Relations, n³ 34, 565-571.

KIVNICK, H.Q. (1982), "Grandparenthood: An overview of meaning and moral health", Gerontologist, $\mathrm{n}^{\circ} 22$, pp. 59-66.

KORNHABER, A. y WOODWARD, K.L. (1981), Grandparents/grandchildren: the vital connection. Garden City, NY, Doubleday.

LANGER, N. (1990), "Grandparents and adult grandchildren: what do they do for one another?" International Journal of Aging and Human Development, vol. 31(2), pp. 101-110.

LERNER, R.M. (1995), "Developing individuals within changing contexts: Implications of developmental contextualism for human development, research, policy and programs", en T.A. Kindermann and J. Valsiner (Eds.), Development of person-context relations, Hillsdale, NJ, Erlbaum.

MATHEWS, S.H. y J. SPREY (1985), “Adolescents' relationships with grandparents: an empirical contribution to conceptual clarification", Journal of Gerontology, n 40, pp. 621-626.

PINAZO, S. (1999a), "Significado social del rol de abuelo", Revista Multidisciplinar de Gerentologia, $\mathrm{n}^{\circ}$ 9, pp. 169-176.

(1999b), "Influencia de los abuelos en la socialización familiar de los nietos. Revisión de la literatura científica", Revista Española de Geriatria y Gerontologia, nº 34 (4), pp. 231-236.

(2002), La imagen de los abuelos a través de los jóvenes ¿Qué piensan los universitarios de la relación con sus abuelos?, en VV.AA., ABUMAR, Jornadas 1998-2001, Madrid, Senda editorial.

RICO, C. (2000), La relación abuelos-nietos al final del milenio, Tesis de Licenciatura, Universidad de Valencia, Dirs.: E. Serra y M.P. Viguer.

RICO, C., SERRA, E. y M. P. VIGUER (2001), Abuelos y nietos: abuelo preferido, abuelo útil, Madrid, Pirámide.

ROBERTO, K.A. y J. STROES (1992), "Grandchildren and grandparents: roles, influences, and relationships", International Journal of Aging and Human Development, Vol. 34, 30, pp. 227 239.

ROBERTSON, J.F. (1976), "Significance of grandparents: perceptions of young adults grandchildren", The Gerontologist, $\mathrm{n}^{\circ} 16(2)$, pp. 137-140.

ROSENMAYR, L. (1977), “The family: a source of hope for the elderly?", en E. Shanas y M.B. Sussman (eds.), Family, bureaucracy and the elderly, Durham, Duke University.

SANCHO, M.T. (coor.) (2000), Informe 2000: Las personas mayores en España, Madrid, IMSERSO. 
RIS

REVISTA INTERNACIONAL DE SOCIOLOCIA

№ 38, MAY0-ACOST0, 2004

SACRAMENTO PINAZO Y JULLÁN MONTORO

SILVERSTEIN, M., R. GIARRUSSO y V. L. BENGTSON (1998), "Intergenerational solidarity and the grandparent role", en M.E. Szinovacs (ed.), Handbook on Grandparenthood, Westport, Conn., Greenwood Press.

SPREY, J. y S. H. MATHEWS (1982), “Contemporary grandparenthood: A systemic transition”, The Annals of the American Academy of Political and Social Science, $\mathrm{n}^{\circ} 464$, pp. 91-103.

THOMAS, J.L. (1986), “Age and sex differences in perceptions of grandparenting", Journal of Gerontology, $\mathrm{n}^{\circ} 41$, pp. 417-423.

TOBÍO, C. y J. A. FERNÁNDEZ-CORDÓN (1999), “Monoparentalidad, trabajo y familia”, Revista Internacional de Socialogia, $\mathrm{n}^{\circ} 22$, pp. 67-97.

TOBÍO, C., J. A. FERNÁNDEZ CORDÓN y M. S. AGULLÓ (1998), Análisis cuantitativo de las estrategias de compatibilización familia-empleo en España, Madrid, Instituto de lã Mujer.

TRIADÓ, C., G. MARTÍNEZ y F. VILLAR (1999), "Relación entre abuelos y sus nietos adolescentes: percepciones, actividades y vínculos", Comunicación presentada al III Congreso Internacional de Psicología y Educación, Santiago de Compostela.

TRIADÓ, C. y F. VILLAR (2000), "El rol de abuelo: cómo perciben los abuelos las relaciones con sus nietos", Revista Española de Geriatria y Gerontologia, n 35, pp. 30-36.

TRIADÓ, C., F. VILLAR, C. SOLÉ, M. J. OSUNA, S. PINAZO y M. CELDRÁN (2004), "Intergenerational relationship in a sample of grandparent-grandchild dyads: do cross-perception of the relationship converge?", International Society for the Study of Behavioural Development Biennial Meeting, Ghent, Belgium.

TYSZKOWA, M. (1991), "Grandparents and grandchildren in Poland", en P.K. Smith (ed.), The psychology of grandparenthood: an international perspective, Londres, Routledge.

(1993), Adolescents' relationship with grandparents: characteristics and developmental transformations, en S. Jackson y H. Rodriguez-Tomé (eds.), Adolescence and its social worlds, Nueva York, Lawrence-Erlbaum Ass.

WILLIAMS, A. y J. F. NUSSBAUM (2001), Intergenerational Communication Across the Life Span, Londres, Lawrence Erlbaum. 